\title{
A Family of Preconditioned Iteratively Regularized Methods For Nonlinear Minimization
}

\author{
Alexandra Smirnova* $\quad$ Rosemary A Renaut ${ }^{\dagger}$
}

March 27, 2008

\begin{abstract}
The preconditioned iteratively regularized Gauss-Newton algorithm for the minimization of general nonlinear functionals was introduced by Smirnova, Renaut and Khan (2007). In this paper, we establish theoretical convergence results for an extended stabilized family of Generalized Preconditioned Iterative methods which includes $\mathcal{M}$-times iterated Tikhonov regularization with line search. Numerical schemes illustrating the theoretical results are also presented.
\end{abstract}

Keywords: Gauss-Newton method, stopping rule, ill-posed problem, regularization.

AMS Subject Classification: 47A52, 65F22, 65J15, 65N21.

\section{Introduction}

Consider a general ill-posed problem of minimizing a nonlinear functional

$$
J(\mathbf{q}):=\left\|F_{\delta}(\mathbf{q})\right\|_{\mathbf{H}_{1}}^{2}
$$

with a noisy operator $F_{\delta}$ mapping between Hilbert spaces $\mathbf{H}$ and $\mathbf{H}_{\mathbf{1}}$, i.e., $F: D(F) \subset \mathbf{H} \rightarrow \mathbf{H}_{\mathbf{1}}$. Notice that this form of the nonlinear functional allows for error both in measurement data, and the forward nonlinear operator used for estimating the measured data. In particular, suppose that $g_{\delta}$ are measured data and $\mathcal{C}(\mathbf{q})$ is a forward operator for obtaining estimates of $g_{\delta}$, then the nonlinear operator in which $\mathcal{C}$ is assumed error free is given by $F_{\delta}=\mathcal{C}-g_{\delta}$. Here we make the more general assumption that $\mathcal{C}$ is also noise contaminated, probably due to a discretization process, and thus the subscript $\delta$ is omitted in the noise free case. When $F_{\delta}$ is Fréchet differentiable in a neighborhood of a minimizer, one of the most used stabilizing numerical algorithms for solving (1.1) is the Iteratively Regularized Gauss-Newton (IRGN) method [BA93]

$$
\mathbf{q}^{(k+1)}=\overline{\mathbf{q}}-\left(F_{\delta}^{\prime *}\left(\mathbf{q}^{(k)}\right) F_{\delta}^{\prime}\left(\mathbf{q}^{(k)}\right)+\tau^{(k)} I\right)^{-1} F_{\delta}^{\prime *}\left(\mathbf{q}^{(k)}\right)\left\{F_{\delta}\left(\mathbf{q}^{(k)}\right)-F_{\delta}^{\prime}\left(\mathbf{q}^{(k)}\right)\left(\mathbf{q}^{(k)}-\overline{\mathbf{q}}\right)\right\}, \quad \overline{\mathbf{q}} \in \mathbf{H} .
$$

\footnotetext{
*Georgia State University, Department of Mathematics and Statistics, Atlanta, GA 30303-3083. Tel: 404-413-6409, Fax: 404-413-6403.

${ }^{\dagger}$ Supported by NSF grants DMS 0513214 and DMS 0652833

¥Arizona State University, Department of Mathematics and Statistics, Tempe, AZ 85287-1804. Tel: 480-965-3795, Fax: 480-965-4160.
} 
The element $\mathbf{q}^{(k+1)}$ in (1.2) has the variational characterization [DES98]

$$
\left\|F_{\delta}\left(\mathbf{q}^{(k)}\right)-F_{\delta}^{\prime}\left(\mathbf{q}^{(k)}\right)\left(\mathbf{q}^{(k)}-\mathbf{q}\right)\right\|_{\mathbf{H}_{\mathbf{1}}}^{2}+\tau^{(k)}\|\mathbf{q}-\overline{\mathbf{q}}\|_{\mathbf{H}}^{2}
$$

For some inverse problems it proves to be extremely beneficial to impose the regularization in an alternative space. This suggests the use of a preconditioned IRGN method [SRK07]

$$
\mathbf{q}^{(k+1)}=\overline{\mathbf{q}}-\left(F_{\delta}^{\prime *}\left(\mathbf{q}^{(k)}\right) F_{\delta}^{\prime}\left(\mathbf{q}^{(k)}\right)+\tau^{(k)} L^{*} L\right)^{-1} F_{\delta}^{\prime *}\left(\mathbf{q}^{(k)}\right)\left\{F_{\delta}\left(\mathbf{q}^{(k)}\right)-F_{\delta}^{\prime}\left(\mathbf{q}^{(k)}\right)\left(\mathbf{q}^{(k)}-\overline{\mathbf{q}}\right)\right\}, \quad \overline{\mathbf{q}} \in \mathbf{H},
$$

with $L \in \mathcal{L}\left(\mathbf{H}, \mathbf{H}_{2}\right)$ and $F_{\delta}^{\prime *}(\cdot) F_{\delta}^{\prime}(\cdot)+\tau L^{*} L$ being invertible for $\tau>0$. Here $\mathbf{q}^{(k+1)}$ has the variational characterization

$$
\left\|F_{\delta}\left(\mathbf{q}^{(k)}\right)-F_{\delta}^{\prime}\left(\mathbf{q}^{(k)}\right)\left(\mathbf{q}^{(k)}-\mathbf{q}\right)\right\|_{\mathbf{H}_{\mathbf{1}}}^{2}+\tau^{(k)}\|L(\mathbf{q}-\overline{\mathbf{q}})\|_{\mathbf{H}_{\mathbf{2}}}^{2}
$$

and the equivalence between the IRGN presented in [SRK07] for the noise free case in $\mathcal{C}$ follows by taking $F_{\delta}=$ $\mathcal{C}-g_{\delta}$. It was shown in [SRK07] that method (1.3) is very effective for the diffusion optical tomography inverse problem, in which the operator $L$ moves regularization from a B-spline coefficient space directly to the physical space. It may also be applied to allow an appropriate weighting on the elements of $\mathbf{q}^{(k)}$ to reflect the differing sensitivities of the operator with respect to different physical components.

Assuming the necessary invertibility conditions on $L^{*} L$, and introducing the notation $T:=\left(L^{*} L\right)^{-1 / 2}$ for self-adjoint operator $T,\left(F_{\delta}^{\prime}\left(\mathbf{q}^{(k)}\right) T\right)^{*}=T F_{\delta}^{\prime *}\left(\mathbf{q}^{(k)}\right),(1.3)$ is rewritten as follows

$$
\mathbf{q}^{(k+1)}=\overline{\mathbf{q}}-T\left[A_{\delta}^{*}\left(\mathbf{q}^{(k)}\right) A_{\delta}\left(\mathbf{q}^{(k)}\right)+\tau^{(k)} I\right]^{-1} A_{\delta}^{*}\left(\mathbf{q}^{(k)}\right)\left\{F_{\delta}\left(\mathbf{q}^{(k)}\right)-F_{\delta}^{\prime}\left(\mathbf{q}^{(k)}\right)\left(\mathbf{q}^{(k)}-\overline{\mathbf{q}}\right)\right\},
$$

where

$$
A_{\delta}\left(\mathbf{q}^{(k)}\right):=F_{\delta}^{\prime}\left(\mathbf{q}^{(k)}\right) T
$$

While scheme (1.4) is, in fact, preconditioned Tikhonov's regularization combined with the Gauss-Newton algorithm, one can use other regularization methods in order to stabilize the Newton step [EHN96]. In particular, replacing $\left[A_{\delta}^{*}\left(\mathbf{q}^{(k)}\right) A_{\delta}\left(\mathbf{q}^{(k)}\right)+\tau^{(k)} I\right]^{-1} A_{\delta}^{*}\left(\mathbf{q}^{(k)}\right)$ in (1.4) by the more general operator $\Phi_{\tau^{(k)}}\left(A_{\delta}\left(\mathbf{q}^{(k)}\right)\right.$, yields

$$
\mathbf{q}^{(k+1)}=\overline{\mathbf{q}}-T \Phi_{\tau^{(k)}}\left(A_{\delta}\left(\mathbf{q}^{(k)}\right)\left\{F_{\delta}\left(\mathbf{q}^{(k)}\right)-F_{\delta}^{\prime}\left(\mathbf{q}^{(k)}\right)\left(\mathbf{q}^{(k)}-\overline{\mathbf{q}}\right)\right\}\right.
$$

The class of methods described by (1.6) includes, amongst others, the $\mathcal{M}$-times iterated preconditioned Tikhonov's method, which is discussed in Section 3. For $T=I$, it has been shown in [BA95] that through their incorporation in the Gauss-Newton process, some of these regularization techniques result in better convergence rates for sufficiently smooth solutions.

Algorithm (1.6) is even more robust and efficient when we introduce a line search procedure with variable step size $\alpha^{(k)}, 0<\alpha \leq \alpha^{(k)} \leq 1$

$$
\mathbf{q}^{(k+1)}=\mathbf{q}^{(k)}+\alpha^{(k)}\left[\overline{\mathbf{q}}-\mathbf{q}^{(k)}-T \Phi_{\tau^{(k)}}\left(A_{\delta}\left(\mathbf{q}^{(k)}\right)\left\{F_{\delta}\left(\mathbf{q}^{(k)}\right)-F_{\delta}^{\prime}\left(\mathbf{q}^{(k)}\right)\left(\mathbf{q}^{(k)}-\overline{\mathbf{q}}\right)\right\}\right] .\right.
$$


The family of methods (1.7) with $T=I$ (no preconditioning) and $\alpha^{(k)}=1$ (no line search) was suggested by A.Bakushinsky in [BA95], and investigated further in [K97], [H97], [BS05], [BKA06], [KN06]. Additional savings may also be realized by reuse of the Jacobian for $\mathcal{M}_{k} \leq \mathcal{M}, \forall k$, inner iteration steps at the outer iteration step $k$ as follows:

For $m=0$ to $\mathcal{M}_{k}-1$ Do

$$
\mathbf{q}^{(k, m+1)}=\mathbf{q}^{(k, m)}+\alpha^{(k, m)}\left[\overline{\mathbf{q}}-\mathbf{q}^{(k, m)}-T \Phi_{\tau^{(k, m)}}\left(A_{\delta}\left(\mathbf{q}^{(k, 0)}\right)\left\{F_{\delta}\left(\mathbf{q}^{(k, m)}\right)-F_{\delta}^{\prime}\left(\mathbf{q}^{(k, 0)}\right)\left(\mathbf{q}^{(k, m)}-\overline{\mathbf{q}}\right)\right\}\right]\right.
$$

\section{End For}

Here the inner steps are initialized with $\mathbf{q}^{(k, 0)}=\mathbf{q}^{\left(k-1, \mathcal{M}_{k-1}\right)}$ and the line search parameter is still constrained by $0<\alpha \leq \alpha^{(k, m)} \leq 1$. An alternative algorithm which uses inner iterations to limit the computational cost uses regularized Landweber iterations for the inner steps, hence avoiding matrix inversion in the inner steps, [K97].

A key to the convergence analysis of the methods presented in this paper, along with the local Lipschitz continuity of $F^{\prime}$, is the modified source condition

$$
\left(L^{*} L\right)(\hat{\mathbf{q}}-\overline{\mathbf{q}})=F^{\prime *}(\hat{\mathbf{q}}) \mathbf{v} \quad \text { for some } \quad \mathbf{v} \in \mathbf{H}_{\mathbf{1}}
$$

This source condition was introduced in [KR93] and used in [SRK07] for the analysis of schemes with preconditioning operator $L$. Here $\hat{\mathbf{q}}$ is a, possibly nonunique, solution to the noise free equation $F(\mathbf{q})=0$. Clearly, for $T=I,(1.9)$ takes the form

$$
\hat{\mathbf{q}}-\overline{\mathbf{q}}=F^{* *}(\hat{\mathbf{q}}) \mathbf{v}, \quad \mathbf{v} \in \mathbf{H}_{1},
$$

which is equivalent to the Hölder source condition

$$
\hat{\mathbf{q}}-\overline{\mathbf{q}}=\left(F^{\prime *}(\hat{\mathbf{q}}) F^{\prime}(\hat{\mathbf{q}})\right)^{1 / 2} \mathbf{w}, \quad \mathbf{w} \in \mathbf{H}
$$

A discussion of the advantages of (1.9) for convergence, and the associated stopping rule, as compared to other adopted convergence conditions and the Lepskij-type a posteriori stopping rule [L90], [BH05], was presented in [SRK07]. Here, we emphasize that our results extend the methods in [SRK07] to both the use of the more general operator $\Phi$, as well as introducing the use of the inner iterations (1.8) for both $T=I$ and the more general $T=\left(L^{*} L\right)^{-1 / 2}$.

The paper is organized as follows: Theorems on the convergence of the iterations (1.7) and (1.8) are presented in Section 2. The resulting numerical schemes are discussed in Section 3.

\section{Convergence Analysis}

We present the details of the basic convergence result for (1.7), followed by the result for (1.8) highlighting only the crucial aspects in which the proofs differ. 
Theorem 1. Assume that

1. $F: D(F) \subset \mathbf{H} \rightarrow \mathbf{H}_{\mathbf{1}}$ with $\mathbf{H}$ and $\mathbf{H}_{\mathbf{1}}$ being Hilbert spaces. The equation $F(\mathbf{q})=0$ is solvable (maybe nonuniquely) and $\hat{\mathbf{q}} \in D(F)$ is a solution.

2. The operators $F$ and $F_{\delta}$ are Fréchet differentiable in $\mathcal{U}_{\eta}(\hat{\mathbf{q}})$, where

$$
\mathcal{U}_{\eta}(\hat{\mathbf{q}})=\{\mathbf{q} \in \mathbf{H}:\|\hat{\mathbf{q}}-\mathbf{q}\| \leq \eta\} \subset D(F)
$$

and $\eta=l \sqrt{\tau^{(0)}}$ with $l$ defined in (2.10) below. There is a positive constant $M$ such that

$$
\left\|F_{\delta}^{\prime}\left(\mathbf{q}_{1}\right)-F_{\delta}^{\prime}\left(\mathbf{q}_{2}\right)\right\| \leq M\left\|\mathbf{q}_{1}-\mathbf{q}_{2}\right\| \quad \text { for any } \quad \mathbf{q}_{1}, \mathbf{q}_{2} \in \mathcal{U}_{\eta}(\hat{\mathbf{q}})
$$

3. $T \in \mathcal{L}(\mathbf{H}, \mathbf{H})$ is a linear self-adjoint operator, $\|T\|:=t$, and for some element $\mathbf{v} \in \mathbf{H}_{1},\|\mathbf{v}\|:=\varepsilon$, the source condition holds

$$
\hat{\mathbf{q}}-\overline{\mathbf{q}}=T A^{*}(\hat{\mathbf{q}}) \mathbf{v}, \quad \text { where } \quad A(\hat{\mathbf{q}}):=F^{\prime}(\hat{\mathbf{q}}) T, \quad \overline{\mathbf{q}} \in \mathbf{H} .
$$

4. The operator $F_{\delta}$ approximates $F$ to the following level of accuracy

$$
\left\|F_{\delta}(\hat{\mathbf{q}})\right\| \leq \delta_{1}, \quad\left\|\left(F^{\prime}(\hat{\mathbf{q}})-F_{\delta}^{\prime}(\hat{\mathbf{q}})\right)^{*} \mathbf{v}\right\| \leq \delta_{2}
$$

5. The regularization sequence $\left\{\tau^{(k)}\right\}$ and the step size sequence $\left\{\alpha^{(k)}\right\}$ satisfy the conditions

$$
\tau^{(k)}>0, \quad \tau^{(k)} \downarrow 0, \quad \sup _{k \in \mathcal{N} \cup\{0\}} \sqrt{\frac{\tau^{(k)}}{\tau^{(k+1)}}}:=d<\infty, \quad 0<\alpha \leq \alpha^{(k)} \leq 1 .
$$

6. For all $G \in \mathcal{L}\left(\mathbf{H}, \mathbf{H}_{\mathbf{1}}\right)$ and $0<\tau$ the regularizer $\Phi_{\tau}$ in (1.7) is constrained by

$$
\begin{aligned}
\left\|\Phi_{\tau}(G) G-I\right\| & \leq C_{1}, \\
\left\|\left(\Phi_{\tau}(G) G-I\right) G^{*}\right\| & \leq C_{2} \sqrt{\tau}, \\
\left\|\Phi_{\tau}(G)\right\| & \leq \frac{C_{3}}{\sqrt{\tau}}, \tau>0 .
\end{aligned}
$$

To simplify the presentation we take $C=\max \left\{C_{1}, C_{2}, C_{3}\right\}$ in the analysis.

7. Iterative process (1.7) is terminated according to the a priori stopping rule

$$
\frac{t \delta_{2}}{\sqrt{\tau^{(k)}}}+\frac{\delta_{1}}{\tau^{(k)}} \leq \rho<\frac{t \delta_{2}}{\sqrt{\tau^{(\mathcal{K})}}}+\frac{\delta_{1}}{\tau^{(\mathcal{K})}}, \quad 0 \leq k<\mathcal{K}=\mathcal{K}\left(\delta_{1}, \delta_{2}\right), \quad \rho>0 .
$$

8. For the constants associated with the operator F and iterations (1.7) the following conditions are fulfilled

$$
\begin{aligned}
& \frac{d-1}{d \alpha}+t^{2} C M \varepsilon+t C \sqrt{2 M(\rho+\varepsilon)} \leq 1 \\
& \frac{\left\|\mathbf{q}^{(0)}-\hat{\mathbf{q}}\right\|}{\sqrt{\tau^{(0)}}} \leq \frac{2 t C(\rho+\varepsilon)}{1-\frac{d-1}{d \alpha}-t^{2} C M \varepsilon}:=l .
\end{aligned}
$$


Then the preconditioned iteratively regularized Gauss-Newton iterations with line search (1.7) satisfy

$$
\frac{\left\|\mathbf{q}^{(k)}-\hat{\mathbf{q}}\right\|}{\sqrt{\tau^{(k)}}} \leq l, \quad k=0,1, \ldots, \mathcal{K}\left(\delta_{1}, \delta_{2}\right)
$$

and

$$
\left\|\mathbf{q}^{(\mathcal{K})}-\hat{\mathbf{q}}\right\|=O(\sqrt{\delta})
$$

where $\delta=\max \left(\delta_{1}, \delta_{2}\right)$.

Proof. Take arbitrary $k<\mathcal{K}\left(\delta_{1}, \delta_{2}\right)$ and suppose that for any $j$ such that $0 \leq j \leq k<\mathcal{K}\left(\delta_{1}, \delta_{2}\right)$ the induction assumption

$$
\sigma^{(j)}:=\frac{\left\|\mathbf{q}^{(j)}-\hat{\mathbf{q}}\right\|}{\sqrt{\tau^{(j)}}} \leq l
$$

holds. Then, one has

$$
\begin{aligned}
\mathbf{q}^{(k+1)}-\hat{\mathbf{q}} & =\alpha^{(k)} \overline{\mathbf{q}}-\hat{\mathbf{q}}+\left(1-\alpha^{(k)}\right) \mathbf{q}^{(k)} \\
& -\alpha^{(k)} T \Phi_{\tau^{(k)}}\left(A_{\delta}\left(\mathbf{q}^{(k)}\right)\right)\left\{F_{\delta}\left(\mathbf{q}^{(k)}\right)-F_{\delta}(\hat{\mathbf{q}})-F_{\delta}^{\prime}\left(\mathbf{q}^{(k)}\right)\left(\mathbf{q}^{(k)}-\hat{\mathbf{q}}\right)-F_{\delta}^{\prime}\left(\mathbf{q}^{(k)}\right)(\hat{\mathbf{q}}-\overline{\mathbf{q}})+F_{\delta}(\hat{\mathbf{q}})\right\} \\
& =J_{1}+J_{2}+J_{3},
\end{aligned}
$$

where

$$
\begin{aligned}
& J_{1}=-\alpha^{(k)} T \Phi_{\tau^{(k)}}\left(A_{\delta}\left(\mathbf{q}^{(k)}\right)\right)\left\{F_{\delta}\left(\mathbf{q}^{(k)}\right)-F_{\delta}(\hat{\mathbf{q}})-F_{\delta}^{\prime}\left(\mathbf{q}^{(k)}\right)\left(\mathbf{q}^{(k)}-\hat{\mathbf{q}}\right)\right\}, \\
& J_{2}=-\alpha^{(k)}\left\{\hat{\mathbf{q}}-\overline{\mathbf{q}}-T \Phi_{\tau^{(k)}}\left(A_{\delta}\left(\mathbf{q}^{(k)}\right)\right) F_{\delta}^{\prime}\left(\mathbf{q}^{(k)}\right)(\hat{\mathbf{q}}-\overline{\mathbf{q}})\right\} \text { and } \\
& J_{3}=\left(1-\alpha^{(k)}\right)\left(\mathbf{q}^{(k)}-\hat{\mathbf{q}}\right)-\alpha^{(k)} T \Phi_{\tau^{(k)}}\left(A_{\delta}\left(\mathbf{q}^{(k)}\right)\right) F_{\delta}(\hat{\mathbf{q}}) .
\end{aligned}
$$

We now estimate $\left\|J_{i}\right\|, i=1,2,3$.

1. By assumption (2.1), (see for example (10.11-10.12) [EHN96]),

$$
\left\|F_{\delta}\left(\mathbf{q}^{(k)}\right)-F_{\delta}(\hat{\mathbf{q}})-F_{\delta}^{\prime}\left(\mathbf{q}^{(k)}\right)\left(\mathbf{q}^{(k)}-\hat{\mathbf{q}}\right)\right\| \leq \frac{M}{2}\left\|\mathbf{q}^{(k)}-\hat{\mathbf{q}}\right\|^{2} .
$$

Thus, from (2.7) one derives

$$
\left\|J_{1}\right\| \leq \frac{\alpha^{(k)} t C}{\sqrt{\tau^{(k)}}} \frac{M}{2}\left\|\mathbf{q}^{(k)}-\hat{\mathbf{q}}\right\|^{2}
$$

2. Source condition (2.2) yields

$$
\hat{\mathbf{q}}-\overline{\mathbf{q}}-T \Phi_{\tau^{(k)}}\left(A_{\delta}\left(\mathbf{q}^{(k)}\right)\right) F_{\delta}^{\prime}\left(\mathbf{q}^{(k)}\right)(\hat{\mathbf{q}}-\overline{\mathbf{q}})=\left[I-T \Phi_{\tau^{(k)}}\left(A_{\delta}\left(\mathbf{q}^{(k)}\right)\right) F_{\delta}^{\prime}\left(\mathbf{q}^{(k)}\right)\right] T A^{*}(\hat{\mathbf{q}}) \mathbf{v}
$$

Adding and subtracting relevant terms, as well as applying (2.2) for $F_{\delta}^{\prime}$, gives

$$
\begin{aligned}
{[I} & \left.-T \Phi_{\tau^{(k)}}\left(A_{\delta}\left(\mathbf{q}^{(k)}\right)\right) F_{\delta}^{\prime}\left(\mathbf{q}^{(k)}\right)\right] T A^{*}(\hat{\mathbf{q}}) \mathbf{v} \\
& =T\left[I-\Phi_{\tau^{(k)}}\left(A_{\delta}\left(\mathbf{q}^{(k)}\right)\right) A_{\delta}\left(\mathbf{q}^{(k)}\right)\right]\left\{\left(A(\hat{\mathbf{q}})-A_{\delta}(\hat{\mathbf{q}})\right)^{*}+\left(A_{\delta}(\hat{\mathbf{q}})-A_{\delta}\left(\mathbf{q}^{(k)}\right)\right)^{*}+A_{\delta}^{*}\left(\mathbf{q}^{(k)}\right)\right\} \mathbf{v},
\end{aligned}
$$


which has the two terms

$$
T\left[I-\Phi_{\tau^{(k)}}\left(A_{\delta}\left(\mathbf{q}^{(k)}\right)\right) A_{\delta}\left(\mathbf{q}^{(k)}\right)\right]\left\{T\left(F^{\prime}(\hat{\mathbf{q}})-F_{\delta}^{\prime}(\hat{\mathbf{q}})\right)^{*}+T\left(F_{\delta}^{\prime}(\hat{\mathbf{q}})-F_{\delta}^{\prime}\left(\mathbf{q}^{(k)}\right)\right)^{*}\right\} \mathbf{v}
$$

and

$$
T\left[I-\Phi_{\tau^{(k)}}\left(A_{\delta}\left(\mathbf{q}^{(k)}\right)\right) A_{\delta}\left(\mathbf{q}^{(k)}\right)\right] A_{\delta}^{*}\left(\mathbf{q}^{(k)}\right) \mathbf{v} .
$$

Hence, assumptions (2.5) and (2.6), with (2.1) and (2.3), imply

$$
\left\|J_{2}\right\| \leq \alpha^{(k)} t^{2} C \delta_{2}+\alpha^{(k)} t^{2} C M\left\|\mathbf{q}^{(k)}-\hat{\mathbf{q}}\right\| \varepsilon+\alpha^{(k)} t C \sqrt{\tau^{(k)}} \varepsilon
$$

3. Finally, by (2.7)

$$
\left\|J_{3}\right\| \leq\left(1-\alpha^{(k)}\right)\left\|\mathbf{q}^{(k)}-\hat{\mathbf{q}}\right\|+\alpha^{(k)} t \frac{C \delta_{1}}{\sqrt{\tau^{(k)}}} .
$$

Summarizing (2.16)-(2.18) one concludes

$$
\begin{aligned}
\left\|\mathbf{q}^{(k+1)}-\hat{\mathbf{q}}\right\| & \leq \frac{\alpha^{(k)} t C M}{2 \sqrt{\tau^{(k)}}}\left\|\mathbf{q}^{(k)}-\hat{\mathbf{q}}\right\|^{2}+\left[1-\alpha^{(k)}+\alpha^{(k)} t^{2} C M \varepsilon\right]\left\|\mathbf{q}^{(k)}-\hat{\mathbf{q}}\right\| \\
& +\alpha^{(k)} t C \sqrt{\tau^{(k)}}\left\{\frac{\delta_{1}}{\tau^{(k)}}+\frac{t \delta_{2}}{\sqrt{\tau^{(k)}}}+\varepsilon\right\} .
\end{aligned}
$$

Because $k<\mathcal{K}\left(\delta_{1}, \delta_{2}\right)$, combining (2.8), (2.19), (2.4) and (2.13),

$$
\sigma^{(k+1)} \leq \frac{\alpha^{(k)} t C M d l^{2}}{2}+\left[1-\alpha^{(k)}+\alpha^{(k)} t^{2} C M \varepsilon\right] d l+\alpha^{(k)} t C d(\rho+\varepsilon) .
$$

Assumptions (2.9) and (2.10), together with (2.20), yield $\sigma^{(k+1)} \leq l$, hence proving inequality (2.11). (2.12) follows from stopping rule (2.8).

\section{Corollary 1. Assume}

$$
\left\|F_{\delta}^{\prime}(\mathbf{q})\right\| \leq N \quad \text { for any } \quad \mathbf{q} \in \mathcal{U}_{\eta}(\hat{\mathbf{q}})
$$

and all conditions of Theorem 1, except

1. stopping rule (2.8) is replaced by the a posteriori stopping rule

$$
\left\|F_{\delta}\left(\mathbf{q}^{(\mathcal{K})}\right)\right\| \leq \mu \sqrt{\delta}<\left\|F_{\delta}\left(\mathbf{q}^{(k)}\right)\right\|, \quad 0 \leq k<\mathcal{K}(\delta), \quad \mu>1, \quad \delta=\max \left\{\delta_{1}, \delta_{2}\right\}, \quad \text { and }
$$

2. (2.9) and (2.10) are respectively replaced by

$$
\frac{d-1}{d \alpha}+t^{2} C M \varepsilon+2 t C \sqrt{\left\{\frac{\left(t \sqrt{\tau^{(0)}}+1\right) N^{2}}{(\mu-1)^{2}}+\frac{M}{2}\right\}} \varepsilon \leq 1,
$$

and

$$
\frac{\left\|\mathbf{q}^{(0)}-\hat{\mathbf{q}}\right\|}{\sqrt{\tau^{(0)}}} \leq \frac{2 t C \varepsilon}{1-\frac{d-1}{d \alpha}-t^{2} C M \varepsilon}:=\tilde{l}
$$


Then the iterations (1.7) satisfy

$$
\frac{\left\|\mathbf{q}^{(k)}-\hat{\mathbf{q}}\right\|}{\sqrt{\tau^{(k)}}} \leq \tilde{l}, \quad k=0,1, \ldots, \mathcal{K}(\delta)
$$

and the sequence $\{\mathcal{K}(\delta)\}$ is admissible.

Proof. From (2.22) it is immediate that for any $k<\mathcal{K}(\delta)$ one has

$$
\mu \sqrt{\delta} \leq\left\|F_{\delta}\left(\mathbf{q}^{(k)}\right)-F_{\delta}(\hat{\mathbf{q}})\right\|+\left\|F_{\delta}(\hat{\mathbf{q}})\right\| \leq N\left\|\mathbf{q}^{(k)}-\hat{\mathbf{q}}\right\|+\delta
$$

and therefore

$$
\mu \sqrt{\delta}-\delta \leq N\left\|\mathbf{q}^{(k)}-\hat{\mathbf{q}}\right\| .
$$

Without loss of generality, one can set $\delta<1$. Thus

$$
\delta \leq \frac{N^{2}\left\|\mathbf{q}^{(k)}-\hat{\mathbf{q}}\right\|^{2}}{(\mu-1)^{2}}
$$

and from (2.19) one obtains

$$
\begin{aligned}
\left\|\mathbf{q}^{(k+1)}-\hat{\mathbf{q}}\right\| & \leq \frac{\alpha^{(k)} t C\left(t \sqrt{\tau^{(0)}}+1\right) N^{2}\left\|\mathbf{q}^{(k)}-\hat{\mathbf{q}}\right\|^{2}}{\sqrt{\tau^{(k)}}(\mu-1)^{2}}+\frac{\alpha^{(k)} t C M}{2 \sqrt{\tau^{(k)}}}\left\|\mathbf{q}^{(k)}-\hat{\mathbf{q}}\right\|^{2} \\
& +\left[1-\alpha^{(k)}+\alpha^{(k)} t^{2} C M \varepsilon\right]\left\|\mathbf{q}^{(k)}-\hat{\mathbf{q}}\right\|+\alpha^{(k)} t C \varepsilon \sqrt{\tau^{(k)}}
\end{aligned}
$$

Using (2.23) and (2.24), result (2.25) now follows. While estimate (2.12) does not follow from stopping rule (2.22), the sequence $\mathcal{K}=\mathcal{K}(\delta)$ is nondecreasing as $\delta \rightarrow 0$. Two cases are possible:

1. $\mathcal{K}(\delta)=\mathcal{K}_{0}$ for any $\delta \leq \delta_{0}$. Then by (2.3) and (2.22) $\mathbf{q}^{\left(\mathcal{K}_{0}\right)}\left(F_{\delta}\right)$ converges to a solution of the equation $F(\mathbf{q})=0$ in the norm of $\mathbf{H}$ as $\delta \rightarrow 0$.

2. $\mathcal{K}(\delta) \rightarrow \infty$ as $\delta \rightarrow 0$. Then

$$
\left\|\mathbf{q}^{(\mathcal{K}(\delta))}-\hat{\mathbf{q}}\right\| \leq \tilde{l} \sqrt{\tau^{(\mathcal{K}(\delta))}} \rightarrow 0 \quad \text { as } \quad \delta \rightarrow 0
$$

Sequence $\{\mathcal{K}(\delta)\}$ is, therefore, admissible.

Remark 1. As opposed to the convergence results for iteratively regularized methods (1.6) with $T=I$, see [BA95], [K97], [BS05], [BKA06], [KN06], in our convergence theorem $\varepsilon$, the norm of $\mathbf{v}$ in the source condition, does not have to be small for inequality (2.9) to be satisfied. Instead, $t^{2} \varepsilon$ with $t:=\|T\|$ must be small. While element $\overline{\mathbf{q}}$ in (1.7) does not need to be close to the solution $\hat{\mathbf{q}}$, condition (2.10) must hold for an appropriate choice of $\tau^{(0)}$.

Remark 2. When the forward operator $F_{\delta}$ is not contaminated by noise, the constant $\delta_{2}$ in (2.3) is zero, and the $a$ priori stopping condition (2.8) simplifies accordingly.

Scheme (1.8), which is just (1.7) when $\mathcal{M}=1$, permits reuse of the operator and has the potential to reduce the overall iteration cost, while possibly increasing the total number of iterations, $k$. In the following note that the $(k, m)$ element of the sequence corresponds to the $i^{\text {th }}$ element where $i=\sum_{p=0}^{k-1} \mathcal{M}_{p}+m$. 
Theorem 2. Assume all conditions of Theorem 1 but with the definitions (2.9) and (2.10) replaced by

$$
\begin{gathered}
\frac{d-1}{d \alpha}+t^{2} C M \varepsilon d^{\mathcal{M}-1}+t C \sqrt{10 M d^{\mathcal{M}-1}(\rho+\varepsilon)} \leq 1, \\
\frac{\left\|\mathbf{q}^{(0,0)}-\hat{\mathbf{q}}\right\|}{\sqrt{\tau^{(0,0)}}} \leq \frac{2 t C(\rho+\varepsilon)}{1-\frac{d-1}{d \alpha}-t^{2} C M \varepsilon d^{\mathcal{M}-1}}:=\hat{l} .
\end{gathered}
$$

Then the iterations (1.8) satisfy

$$
\frac{\left\|\mathbf{q}^{(k, m)}-\hat{\mathbf{q}}\right\|}{\sqrt{\tau^{(k, m)}}} \leq \hat{l}, \quad \sum_{p=0}^{k-1} \mathcal{M}_{p}+m=0,1, \ldots, \mathcal{K}\left(\delta_{1}, \delta_{2}\right) .
$$

Proof. The result follows very similarly to the proof of Theorem 1. Assume that for the first $i$ elements of the sequence,

$$
\sigma^{(k, m)}:=\frac{\left\|\mathbf{q}^{(k, m)}-\hat{\mathbf{q}}\right\|}{\sqrt{\tau^{(k, m)}}} \leq \hat{l}, \quad 0 \leq \sum_{p=0}^{k-1} \mathcal{M}_{p}+m \leq i<\mathcal{K}\left(\delta_{1}, \delta_{2}\right) .
$$

The $(i+1)^{\text {st }}$ element of the sequence is $\mathbf{q}^{(k, m+1)}$ (if $m<\mathcal{M}_{k}$ ), or $\mathbf{q}^{(k+1,0)}$ (if $m=\mathcal{M}_{k}$ ). Let $m<\mathcal{M}_{k}$. Expression (2.14) is replaced by

$$
\begin{aligned}
\mathbf{q}^{(k, m+1)}-\hat{\mathbf{q}} & =\alpha^{(k, m)} \overline{\mathbf{q}}-\hat{\mathbf{q}}+\left(1-\alpha^{(k, m)}\right) \mathbf{q}^{(k, m)}-\alpha^{(k, m)} T \Phi_{\tau^{(k, m)}}\left(A_{\delta}\left(\mathbf{q}^{(k, 0)}\right)\right)\left\{F_{\delta}\left(\mathbf{q}^{(k, m)}\right)\right. \\
& \left.-F_{\delta}(\hat{\mathbf{q}})-F_{\delta}^{\prime}\left(\mathbf{q}^{(k, 0)}\right)\left(\mathbf{q}^{(k, m)}-\hat{\mathbf{q}}\right)-F_{\delta}^{\prime}\left(\mathbf{q}^{(k, 0)}\right)(\hat{\mathbf{q}}-\overline{\mathbf{q}})+F_{\delta}(\hat{\mathbf{q}})\right\}
\end{aligned}
$$

Notice now that

$$
\begin{aligned}
J_{1} & =-\alpha^{(k, m)} T \Phi_{\tau^{(k, m)}}\left(A_{\delta}\left(\mathbf{q}^{(k, 0)}\right)\right)\left\{F_{\delta}\left(\mathbf{q}^{(k, m)}\right)-F_{\delta}(\hat{\mathbf{q}})\right. \\
& \left.-F_{\delta}^{\prime}\left(\mathbf{q}^{(k, m)}\right)\left(\mathbf{q}^{(k, m)}-\hat{\mathbf{q}}\right)+\left(F_{\delta}^{\prime}\left(\mathbf{q}^{(k, m)}\right)-F_{\delta}^{\prime}\left(\mathbf{q}^{(k, 0)}\right)\right)\left(\mathbf{q}^{(k, m)}-\hat{\mathbf{q}}\right)\right\}
\end{aligned}
$$

Thus

$$
\begin{aligned}
\left\|J_{1}\right\| & \leq \frac{\alpha^{(k, m)} t C M}{2 \sqrt{\tau^{(k, m)}}}\left\|\mathbf{q}^{(k, m)}-\hat{\mathbf{q}}\right\|^{2}+\frac{\alpha^{(k, m)} t C M}{\sqrt{\tau^{(k, m)}}}\left\|\mathbf{q}^{(k, m)}-\hat{\mathbf{q}}\right\|\left\|\mathbf{q}^{(k, m)}-\mathbf{q}^{(k, 0)}\right\| \\
& \leq \frac{3 \alpha^{(k, m)} t C M}{2 \sqrt{\tau^{(k, m)}}}\left\|\mathbf{q}^{(k, m)}-\hat{\mathbf{q}}\right\|^{2}+\frac{\alpha^{(k, m)} t C M}{\sqrt{\tau^{(k, m)}}}\left\|\mathbf{q}^{(k, 0)}-\hat{\mathbf{q}}\right\|\left\|\mathbf{q}^{(k, m)}-\hat{\mathbf{q}}\right\| .
\end{aligned}
$$

The bound of $\left\|J_{2}\right\|$ is obtained as for (2.17) yielding

$$
\left\|J_{2}\right\| \leq \alpha^{(k, m)} t^{2} C \delta_{2}+\alpha^{(k, m)} t^{2} C M\left\|\mathbf{q}^{(k, 0)}-\hat{\mathbf{q}}\right\| \varepsilon+\alpha^{(k, m)} t C \sqrt{\tau^{(k, m)}} \varepsilon,
$$

and we also immediately obtain

$$
\left\|J_{3}\right\| \leq\left(1-\alpha^{(k, m)}\right)\left\|\mathbf{q}^{(k, m)}-\hat{\mathbf{q}}\right\|+\alpha^{(k, m)} t \frac{C \delta_{1}}{\sqrt{\tau^{(k, m)}}} .
$$

Therefore

$$
\begin{aligned}
\| \mathbf{q}^{(k, m+1)} & -\hat{\mathbf{q}}\left\|\leq \frac{3 \alpha^{(k, m)} t C M}{2 \sqrt{\tau^{(k, m)}}}\right\| \mathbf{q}^{(k, m)}-\hat{\mathbf{q}}\left\|^{2}+\left[1-\alpha^{(k, m)}\right]\right\| \mathbf{q}^{(k, m)}-\hat{\mathbf{q}}\left\|+\alpha^{(k, m)} t^{2} C M \varepsilon\right\| \mathbf{q}^{(k, 0)}-\hat{\mathbf{q}} \| \\
& +\alpha^{(k, m)} t C \sqrt{\tau^{(k, m)}}\left\{\frac{\delta_{1}}{\tau^{(k, m)}}+\frac{t \delta_{2}}{\sqrt{\tau^{(k, m)}}}+\varepsilon\right\}+\frac{\alpha^{(k, m)} t C M}{\sqrt{\tau^{(k, m)}}}\left\|\mathbf{q}^{(k, m)}-\hat{\mathbf{q}}\right\|\left\|\mathbf{q}^{(k, 0)}-\hat{\mathbf{q}}\right\|(2.37)
\end{aligned}
$$


By the induction, and using result $d \leq d^{\mathcal{M}}$ in order to combine first and fith terms of (2.37), one arrives at

$$
\sigma^{(k, m+1)} \leq \frac{5 \alpha^{(k, m)} t C M d^{\mathcal{M}} \hat{l}^{2}}{2}+\left[d-d \alpha^{(k)}+\alpha^{(k, m)} t^{2} C M \varepsilon d^{\mathcal{M}}\right] \hat{l}+\alpha^{(k, m)} t C d(\rho+\varepsilon),
$$

and the result follows. Finally, if $m=\mathcal{M}_{k}$, the result holds by Theorem 1 .

\section{Discussion of the Numerical Schemes}

In this section we consider examples of generating operators $\Phi_{\tau}(G), G \in \mathcal{L}\left(\mathbf{H}, \mathbf{H}_{\mathbf{1}}\right)$, motivated by different regularization techniques merged with Gauss-Newton iterations.

Example 1. From (1.3) and (1.4) it is clear that for

$$
\Phi_{\tau}(G):=\left[G^{*} G+\tau I\right]^{-1} G^{*}
$$

algorithm (1.7) takes the form:

$$
\mathbf{q}^{(k+1)}=\mathbf{q}^{(k)}+\alpha^{(k)} \mathbf{p}^{(k)},
$$

where the search direction $\mathbf{p}^{(k)}$ is the solution of

$$
\left[F_{\delta}^{\prime *}\left(\mathbf{q}^{(k)}\right) F_{\delta}^{\prime}\left(\mathbf{q}^{(k)}\right)+\tau^{(k)} L^{*} L\right] \mathbf{p}^{(k)}=-\left[F_{\delta}^{\prime *}\left(\mathbf{q}^{(k)}\right) F_{\delta}\left(\mathbf{q}^{(k)}\right)+\tau^{(k)} L^{*} L\left(\mathbf{q}^{(k)}-\overline{\mathbf{q}}\right)\right] .
$$

This line search algorithm with search direction obtained from (3.3) was introduced and analyzed in [SRK07]. Here, we extend its use by adopting the appropriately initialized inner iterations (1.8)

For $m=0$ to $\mathcal{M}_{k}-1$ Do

$\mathbf{q}^{(k, m+1)}=\mathbf{q}^{(k, m)}-\alpha^{(k, m)}\left[F_{\delta}^{\prime *}\left(\mathbf{q}^{(k)}\right) F_{\delta}^{\prime}\left(\mathbf{q}^{(k)}\right)+\tau^{(k, m)} L^{*} L\right]^{-1}\left[F_{\delta}^{\prime *}\left(\mathbf{q}^{(k)}\right) F_{\delta}\left(\mathbf{q}^{(k, m)}\right)+\tau^{(k, m)} L^{*} L\left(\mathbf{q}^{(k, m)}-\overline{\mathbf{q}}\right)\right]$

\section{End For}

Each inner update requires a system solve with system matrix $\left[G^{*} G+\tau^{(k, m)} I\right]$ which can be obtained cheaply provided that a method such as preconditioned conjugate gradients is adopted at every step and for $\mathcal{M}_{k}>1$ the solution is initialized with a good initial starting value, presumably the last value from the previous iteration.

Example 2. In order to further stabilize the Gauss-Newton step, we may make use of $\mathcal{M}$-times repeated Tikhonov regularization, ([BA95], Section 5.1 [EHN96]). The search direction $\mathbf{p}^{(k)}$ in (3.2) is computed as the result of inner iterations in which the solution is prevented from stepping too far from the previous mapped value of $\mathbf{q}^{(k)}$.

Calculate $\mathbf{p}^{(k, 1)}$ by (3.3)

For $\quad m=1$ to $\mathcal{M}-1$ Do

$$
\mathbf{p}^{(k, m+1)}=-\left[F_{\delta}^{\prime *}\left(\mathbf{q}^{(k)}\right) F_{\delta}^{\prime}\left(\mathbf{q}^{(k)}\right)+\tau^{(k)} L^{*} L\right]^{-1}\left[F_{\delta}^{\prime *}\left(\mathbf{q}^{(k)}\right) F_{\delta}\left(\mathbf{q}^{(k)}\right)-\tau^{(k)} L^{*} L \mathbf{p}^{(k, m)}\right]
$$

\section{End For}

$\mathbf{q}^{(k+1)}=\mathbf{q}^{(k)}+\alpha^{(k)} \mathbf{p}^{(k, \mathcal{M})}$ 
Practically the algorithm is implemented as given, but to see how it differs from (3.4) notice that the inner updates, for $m<\mathcal{M}-1$, can be written in the equivalent form

$$
\mathbf{q}^{(k+1, m+1)}=\mathbf{q}^{(k)}-\left[F_{\delta}^{\prime *}\left(\mathbf{q}^{(k)}\right) F_{\delta}^{\prime}\left(\mathbf{q}^{(k)}\right)+\tau^{(k)} L^{*} L\right]^{-1}\left[F_{\delta}^{\prime *}\left(\mathbf{q}^{(k)}\right) F_{\delta}\left(\mathbf{q}^{(k)}\right)+\tau^{(k)} L^{*} L\left(\mathbf{q}^{(k)}-\mathbf{q}^{(k+1, m)}\right)\right]
$$

with the line search parameter $\alpha^{(k)}$ introduced for $m=\mathcal{M}-1$.

We now show that this iteration is equivalent to (1.7) for the operator

$$
\Phi_{\tau}(G):=\sum_{i=0}^{\mathcal{M}-1} \tau^{i}\left[G^{*} G+\tau I\right]^{-(i+1)} G^{*} .
$$

Introduce the notation

$$
D_{k}:=\left[F_{\delta}^{\prime *}\left(\mathbf{q}^{(k)}\right) F_{\delta}^{\prime}\left(\mathbf{q}^{(k)}\right)+\tau^{(k)} B\right], \quad \mathbf{g}_{k}:=F_{\delta}^{\prime *}\left(\mathbf{q}^{(k)}\right) F_{\delta}\left(\mathbf{q}^{(k)}\right), \quad B=L^{*} L
$$

then

$$
\mathbf{p}^{(k, m+1)}=\tau^{(k)} D_{k}^{-1} B \mathbf{p}^{(k, m)}-D_{k}^{-1} \mathbf{g}_{k}, \quad m \geq 1
$$

and

$$
D_{k}^{-1} B=\left[F_{\delta}^{\prime *}\left(\mathbf{q}^{(k)}\right) F_{\delta}^{\prime}\left(\mathbf{q}^{(k)}\right)+\tau^{(k)} B\right]^{-1} B=T\left[A_{\delta}^{*}\left(\mathbf{q}^{(k)}\right) A_{\delta}\left(\mathbf{q}^{(k)}\right)+\tau^{(k)} I\right]^{-1} T^{-1}:=Y_{k} .
$$

Noticing from (3.3) that we can define $\mathbf{p}^{(k, 0)}=\overline{\mathbf{q}}-\mathbf{q}^{(k)}$, one obtains

$$
\begin{gathered}
\mathbf{p}^{(k, \mathcal{M})}=\left(\tau^{(k)}\right)^{\mathcal{M}} Y_{k}^{\mathcal{M}}\left(\overline{\mathbf{q}}-\mathbf{q}^{(k)}\right)-\sum_{i=0}^{\mathcal{M}-1}\left(\tau^{(k)}\right)^{i} Y_{k}^{i} D_{k}^{-1} \mathbf{g}_{k} . \\
Y_{k}^{\mathcal{M}}=T \Lambda_{k}^{-\mathcal{M}} T^{-1}, \quad \text { where } \quad \Lambda_{k}:=A_{\delta}^{*}\left(\mathbf{q}^{(k)}\right) A_{\delta}\left(\mathbf{q}^{(k)}\right)+\tau^{(k)} I .
\end{gathered}
$$

Identity (3.11) yields

$$
\begin{aligned}
\mathbf{p}^{(k, \mathcal{M})} & =\left(\tau^{(k)}\right)^{\mathcal{M}} T \Lambda_{k}^{-\mathcal{M}} T^{-1}\left(\overline{\mathbf{q}}-\mathbf{q}^{(k)}\right)-\sum_{i=0}^{\mathcal{M}-1}\left(\tau^{(k)}\right)^{i} T \Lambda_{k}^{-(i+1)} T F_{\delta}^{\prime *}\left(\mathbf{q}^{(k)}\right) F_{\delta}\left(\mathbf{q}^{(k)}\right) \\
& =\left(\tau^{(k)}\right)^{\mathcal{M}} T \Lambda_{k}^{-\mathcal{M}} T^{-1}\left(\overline{\mathbf{q}}-\mathbf{q}^{(k)}\right)-\sum_{i=0}^{\mathcal{M}-1}\left(\tau^{(k)}\right)^{i} T \Lambda_{k}^{-(i+1)} A_{\delta}^{*}\left(\mathbf{q}^{(k)}\right) F_{\delta}^{\prime}\left(\mathbf{q}^{(k)}\right)\left(\mathbf{q}^{(k)}-\overline{\mathbf{q}}\right) \\
& -\sum_{i=0}^{\mathcal{M}-1}\left(\tau^{(k)}\right)^{i} T \Lambda_{k}^{-(i+1)} A_{\delta}^{*}\left(\mathbf{q}^{(k)}\right)\left\{F_{\delta}\left(\mathbf{q}^{(k)}\right)-F_{\delta}^{\prime}\left(\mathbf{q}^{(k)}\right)\left(\mathbf{q}^{(k)}-\overline{\mathbf{q}}\right)\right\} .
\end{aligned}
$$

But now, by induction, the first two terms in (3.10) simplify to $\overline{\mathbf{q}}-\mathbf{q}^{(k)}$, and (3.10) becomes

$$
\mathbf{p}^{(k, \mathcal{M})}=\overline{\mathbf{q}}-\mathbf{q}^{(k)}-T \sum_{i=0}^{\mathcal{M}-1}\left(\tau^{(k)}\right)^{i} \Lambda_{k}^{-(i+1)} A_{\delta}^{*}\left(\mathbf{q}^{(k)}\right)\left\{F_{\delta}\left(\mathbf{q}^{(k)}\right)-F_{\delta}^{\prime}\left(\mathbf{q}^{(k)}\right)\left(\mathbf{q}^{(k)}-\overline{\mathbf{q}}\right)\right\},
$$

from which (3.7) follows. It remains to verify that (3.7) meets conditions (2.5)-(2.7) of Theorem 1.

1.

$$
\left\|\Phi_{\tau}(G) G-I\right\|=\left\|\sum_{i=0}^{\mathcal{M}-1} \tau^{i}\left[G^{*} G+\tau I\right]^{-(i+1)} G^{*} G-I\right\| \leq \sup _{\lambda \in[0, \infty)}\left|\sum_{i=0}^{\mathcal{M}-1} \tau^{i}(\lambda+\tau)^{-(i+1)} \lambda-1\right|
$$




$$
=\sup _{\lambda \in[0, \infty)}\left[\frac{\tau}{\lambda+\tau}\right]^{\mathcal{M}} \leq 1
$$

2.

$$
\begin{gathered}
\left\|\left(\Phi_{\tau}(G) G-I\right) G^{*}\right\|=\left\|\left(\sum_{i=0}^{\mathcal{M}-1} \tau^{i}\left[G^{*} G+\tau I\right]^{-(i+1)} G^{*} G-I\right) G^{*}\right\| \leq \sup _{\lambda \in[0, \infty)}\left[\frac{\tau}{\lambda+\tau}\right]^{\mathcal{M}} \sqrt{\lambda} \\
=\frac{\tau^{\frac{1}{2}}(2 \mathcal{M}-1)^{\mathcal{M}-\frac{1}{2}}}{(2 \mathcal{M})^{\mathcal{M}}} \leq \sqrt{\frac{\tau}{2 \mathcal{M}}}
\end{gathered}
$$

3. Finally,

$$
\begin{gathered}
\left\|\Phi_{\tau}(G)\right\|=\left\|\left|\sum_{i=0}^{\mathcal{M}-1} \tau^{i}\left[G^{*} G+\tau I\right]^{-(i+1)} G^{*} \| \leq \sup _{\lambda \in[0, \infty)}\right| \sum_{i=0}^{\mathcal{M}-1} \tau^{i}(\lambda+\tau)^{-(i+1)} \sqrt{\lambda} \mid\right. \\
=\sup _{\lambda \in[0, \infty)} \frac{\sqrt{\lambda}}{\lambda+\tau} \sum_{i=0}^{\mathcal{M}-1}\left[\frac{\tau}{\lambda+\tau}\right]^{i}=\sup _{\lambda \in[0, \infty)}\left\{\sum_{i=0}^{\mathcal{M}-1}\left[1-\frac{\lambda}{\lambda+\tau}\right]^{i} \sqrt{\frac{\lambda}{\lambda+\tau}}\right\} \frac{1}{\sqrt{\lambda+\tau}} .
\end{gathered}
$$

To complete the estimate, we apply Lemma 3.1 [BS05]

$$
\sup _{a \in[0,1]} \sum_{i=0}^{P}(1-a)^{i} \sqrt{a} \leq \sqrt{2(P+1)}
$$

With $a=\frac{\lambda}{\lambda+\tau}$ and $P=\mathcal{M}-1$ one obtains $\left\|\Phi_{\tau}(G)\right\| \leq \sqrt{\frac{2 \mathcal{M}}{\tau}}$.

Example 3. A modification of (3.5) replaces the dependence of the iterative step in (3.5) on $\tau^{(k)}$ by the constant parameter $\sigma>0$,

$$
\left[F_{\delta}^{\prime *}\left(\mathbf{q}^{(k)}\right) F_{\delta}^{\prime}\left(\mathbf{q}^{(k)}\right)+\sigma L^{*} L\right] \mathbf{p}^{(k, m+1)}=-F_{\delta}^{\prime *}\left(\mathbf{q}^{(k)}\right) F_{\delta}\left(\mathbf{q}^{(k)}\right)+\sigma L^{*} L \mathbf{p}^{(k, m)}
$$

It is immediate by the derivation of (3.12) that in this case

$$
\mathbf{p}^{(k, \mathcal{M})}=\overline{\mathbf{q}}-\mathbf{q}^{(k)}-T \sum_{i=0}^{\mathcal{M}_{k}-1} \sigma^{i}\left[A_{\delta}^{*}\left(\mathbf{q}^{(k)}\right) A_{\delta}\left(\mathbf{q}^{(k)}\right)+\sigma I\right]^{-(i+1)} A_{\delta}^{*}\left(\mathbf{q}^{(k)}\right)\left\{F_{\delta}\left(\mathbf{q}^{(k)}\right)-F_{\delta}^{\prime}\left(\mathbf{q}^{(k)}\right)\left(\mathbf{q}^{(k)}-\overline{\mathbf{q}}\right)\right\} .
$$

Now taking $\mathcal{M}_{k} \rightarrow \infty$ as $k \rightarrow \infty$ to satisfy the condition $\tau^{(k)} \rightarrow 0$ for $\tau^{(k)}:=1 / \mathcal{M}_{k}$, gives (1.7) with operator

$$
\Phi_{\tau}(G):=\sum_{i=0}^{\frac{1}{\tau}-1} \sigma^{i}\left[G^{*} G+\sigma I\right]^{-(i+1)} G^{*}
$$

Again, it remains to verify conditions (2.5)-(2.7).

1. For (2.5) $\left\|\Phi_{\tau}(G) G-I\right\| \leq \sup _{\lambda \in[0, \infty)}\left[\frac{\sigma}{\lambda+\sigma}\right]^{\frac{1}{\tau}} \leq 1$.

2. For (2.6) $\left\|\left(\Phi_{\tau}(G) G-I\right) G^{*}\right\| \leq \sup _{\lambda \in[0, \infty)}\left[\frac{\sigma}{\lambda+\sigma}\right]^{\frac{1}{\tau}} \sqrt{\lambda}=\frac{\sigma^{\frac{1}{2}}(2-\tau)^{\frac{1}{\tau}-\frac{1}{2}}}{\tau^{-\frac{1}{2}} 2^{\frac{1}{\tau}}} \leq \sqrt{\frac{\sigma \tau}{2}}$, provided $\tau<2$, which is immediate for $\mathcal{M} \geq 1$.

3. To obtain (2.7) apply again Lemma 3.1 [BS05] with $a=\frac{\lambda}{\lambda+\sigma}$ and $P=\frac{1}{\tau}-1$, yielding $\left\|\Phi_{\tau}(G)\right\| \leq \sqrt{\frac{2}{\tau \sigma}}$. 
Remark 3. From Example 3, it is now evident that introducing a line search parameter in (3.6)

$$
\mathbf{q}^{(k+1, m)}=\mathbf{q}^{(k)}+\alpha^{(k, m)} \mathbf{p}^{(k, m)},
$$

replaces $\sigma$ on the right hand side in (3.5) by $\sigma^{(k, m)}=\sigma / \alpha^{(k, m)}$. An equivalent modification occurs in (3.5), and the distinction between (3.12) and (3.13) is lost. Moreover, it is not possible to reformulate the new parameter dependent scheme in terms of (1.7), and we conclude that line searching in the inner iterations is not feasible unless we utilize (1.8).

\section{Conclusions}

Theoretical convergence results and numerical implementation of a family of preconditioned iteratively regularized Gauss-Newton schemes for solution of general ill-posed nonlinear minimization have been presented. These extend the initial theory of [SRK07] for application of preconditioning to a larger class of iterative schemes with line search, and have potential application in a wide variety of ill-posed problems.

\section{References}

[BA93] Bakushinsky, A. B., Iterative methods for nonlinear operator equations without regularity, new approach, Dokl. Russian Acad. Sci. 330 282-284, (1993).

[BA95] Bakushinsky, A. B., Iterative methods without saturation for solving degenerate nonlinear operator equations, Dokl. Russian Acad. Sci. 334 7-8, (1995).

[BS05] Bakushinsky, A. B. and Smirnova, A. On application of generalized discrepancy principle to iterative methods for nonlinear ill-posed problems, Numerical Functional Analysis and Optimization, 26, N1, 35-48, (2005).

[BH05] Bauer, F. and Hohage, T. A Lepskij-type stopping rule for regularized Newton methods, Inverse Problems, 21, 1975-1991, (2005).

[BKA06] Burger M., and Kaltenbacher B., Regularizing NewtonKaczmarz Methods for Nonlinear Ill-Posed Problems, SIAM J. Num Anal., 44, N1, 153-182, (2006).

[DES98] Deuflhard, P., Engl, H.W. and Scherzer, O. A convergence analysis of iterative methods for the solution of nonlinear ill-posed problems under affinely invariant conditions, Inv. Probl., 14, 1081-1106, (1998).

[EHN96] Engl, H., Hanke, M. and Neubauer, A. Regularization of Inverse Problems, Kluwer Academic Publisher, Dordecht, Boston, London, (1996).

[H97] Hohage, T. Logarithmic convergence rates of the iteratively regularized Gauss-Newton method for an inverse potential and inverse scattering problem, Inverse Problems, 13, 1279-1299, (1997). 
[K97] Kaltenbacher, B., Some Newton-type methods for the regularization of nonlinear ill-posed problems, Inverse Problems, 13, 729-753, (1997).

[KN06] Kaltenbacher, B. and Neubauer, A. Convergence of projected iterative regularization methods for nonlinear problems with smooth solutions. Inverse Problems, 22, N3, 1105-1119, (2006).

[KR93] Kunisch K. and Ring N. Regularization of nonlinear illposed problems with closed operators. Numerical Functional Analysis and Optimization, 14, 389-404, (1993).

[L90] Lepskij, O.V. On a problem of adaptive estimation in Gaussian white noise, Theory Probab. Appl., 35, 454-466, (1990).

[SRK07] Smirnova, A.B., Renaut R.A., and Khan, T. Convergence and application of a modified iteratively regularized GaussNewton algorithm. Inverse Problems, 23, N4, 1547-1563 (2007). 